\title{
TUBERCULOSIS PERITONEAL EN UNA PACIENTE CON ASCITIS Y MASA ANEXIAL. REPORTE DE UN CASO
}

\section{Peritoneal tuberculosis in a patient with ascites and an adnexal mass. A case report}

Germán García, M.D. *, Andrés José García, M.D. **, Elvia Inés Goez, M.D. ***, Clara Inés Saldarriaga, M.D. ****

Recibido: febrero 17/2006 - Revisado: marzo 21/2006 - Aceptado: marzo 30/2006

\section{RESUMEN}

Objetivo: presentar el cuadro clínico y de laboratorio de una paciente con tuberculosis peritoneal. La tuberculosis puede afectar diferentes órganos con presentaciones clínicas que simulan otras patologías. ${ }^{1}$ Presentamos el caso de una paciente con ascitis y masa anexial diagnosticada por tomografía y con CA 125 elevado. Se realizó laparotomía exploradora con la impresión diagnóstica de tumor de ovario, encontrando adherencias y siembras peritoneales. La patología informó inflamación granulomatosa crónica compatible con tuberculosis.

Palabras clave: tuberculosis, ascitis, quistes ováricos, enfermedades de los anexos, antígeno Ca-125

\section{SUMMARY}

Objective: Describing the clinical picture and laboratory results of a patient suffering from peritoneal tuberculosis.

* Ginecólogo-Oncólogo, Profesor Oncología Ginecológica, Universidad de Antioquia. Hospital Universitario San Vicente de Paúl. Correo electrónico: gegar65@yahoo.com

** Profesor de Medicina Interna, Universidad de Antioquia. Hospital Universitario San Vicente de Paúl.

*** Patóloga Oncóloga, Hospital Universitario San Vicente de Paúl.

**** Residente I, Medicina Interna, Universidad de Antioquía.
Tuberculosis can affect different organs, having different presentations mimicking other diseases. ${ }^{1}$ We present the case of a 24 -year-old patient suffering from ascites and adnexal mass (abdominal CT scan revealed an ovarian cyst) accompanied by high CA-125 antigen titres. Exploratory laparotomy revealed strictures and peritoneal seeds. Pathology revealed chronic granulomatous lesions suggesting tuberculosis

Key words: tuberculosis, ascites, ovarian cyst, CA 125 antigen

\section{CASO CLÍNICO}

Paciente de sexo femenino, natural y residente en Quibdó, capital del departamento de Chocó, Colombia, sin antecedentes patológicos de importancia; consulta por astenia, adinamia, pérdida de peso no cuantificada, fiebre subjetiva e incremento del perímetro abdominal de un mes de evolución. Al examen físico se observa caquéctica y con palidez mucocutánea generalizada, presentaba ascitis y se palpaba al tacto vaginal una masa anexial derecha. Los exámenes de laboratorio de ingreso mostraron hemoglobina 9,9 mg/dl, hematocrito 30,4\%, leucocitos $6.800 / \mathrm{mm}^{3}$ con $72 \%$ de neutrófilos y $14 \%$ de linfocitos, plaquetas $610.000 / \mathrm{mm}^{3}$. Se realiza para- 
centesis diagnóstica encontrando $\mathrm{pH} 8$, Densidad $1.034 \mathrm{mg} / \mathrm{dl}$, proteínas $6,2 \mathrm{~g} / \mathrm{l}$, leucocitos $7 / \mathrm{mm}^{3}$, polimorfonucleares $4 / \mathrm{mm}^{3}$, monomucleares $3 / \mathrm{mm}^{3}$, albúmina en líquido peritoneal 2,2 g/l (sérica 3,3 $\mathrm{g} / \mathrm{l})$. La coloración para bacilos ácido alcohol-resistentes que se reportó como negativa. Se descartó infección por VIH mediante realización de prueba de ELISA. Posteriormente se realizó ecografía transvaginal y TAC de abdomen, que reportaron lesión adyacente en anexo derecho de 32 x $22 \mathrm{~mm}$ con características de quiste complejo de ovario (Figura 1). Se solicita $\mathrm{Ca}-125$, encontrándose aumentado (233 U/mL) con referencia a los valores normales $(0-35)$, se solicitan además rayos x de tórax, en los que se observa aumento de la densidad y tamaño de los hilios pulmonares asociado a imágenes nodulares en relación con calcificaciones cicatriciales ganglionares. Dos días después del ingreso la paciente fue llevada a cirugía con impresión diagnóstica de tumor de ovario y carcinomatosis peritoneal, le realizaron laparotomía exploradora, encontrando pelvis con múltiples adherencias (pelvis congelada), con implantes miliares peritoneales menores de $1 \mathrm{~cm}$ y 4.600 cc de líquido ascítico. No se observaron masas en ovarios. Se tomaron biopsias de peritoneo para patología y citología que informaron abundantes granulomas con necrosis de caseificación y células gigantes multinucleadas, hallazgos sugestivos de tuberculosis (Figura 2). Esta fue confirmada por una coloración de Ziehl Nielsen positiva para bacilos ácidoalcohol resistentes en escasa cantidad (Figura 3), no se

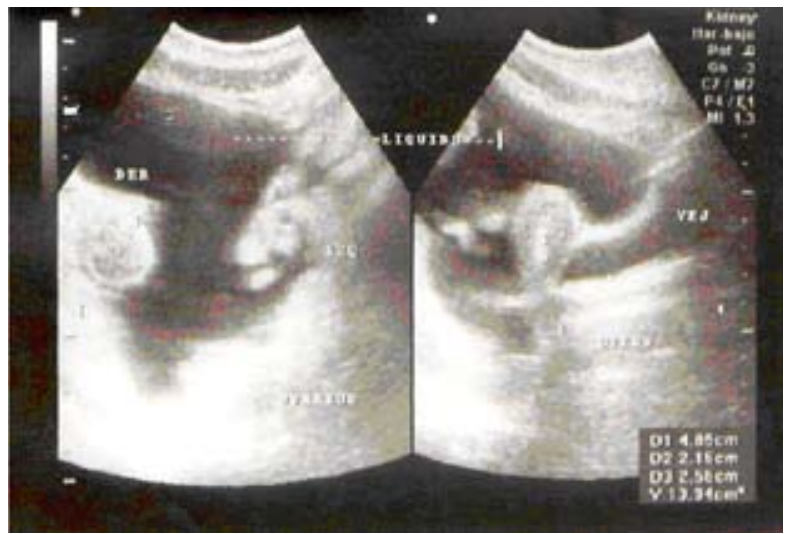

Figura 1. Ecografía

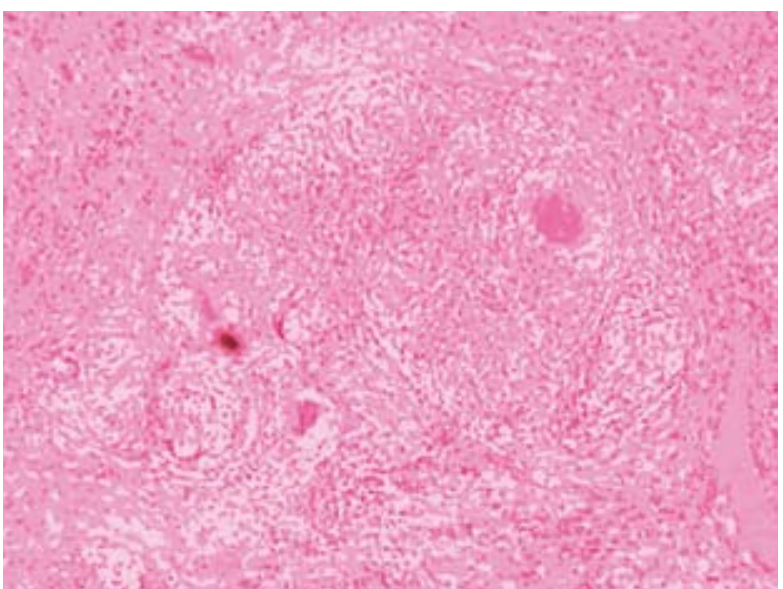

Figura 2. Granulomas tuberculosos, coloración hematoxilina -eosina

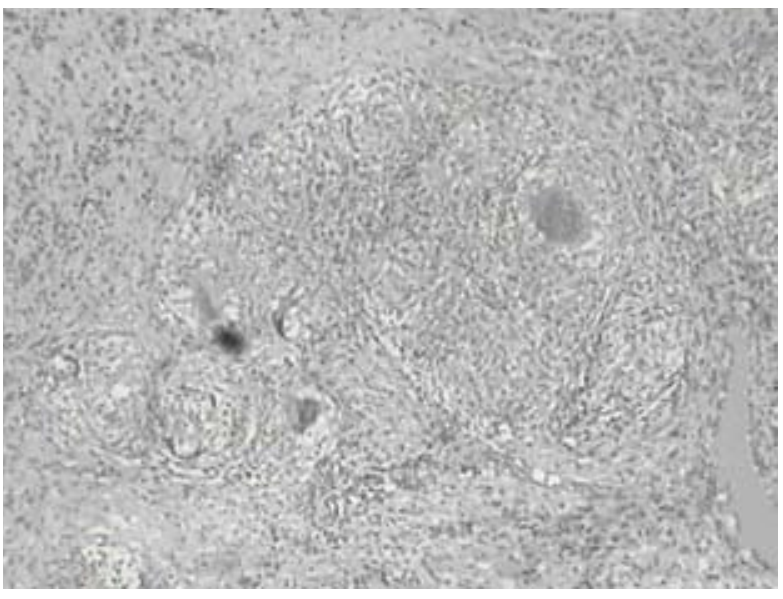

Figura 3. Granulomas tuberculosos, coloración Ziehl Nielsen

observaron criterios de malignidad. Adicionalmente informan adenosin deaminasa en líquido peritoneal $106 \mathrm{u} / \mathrm{l}$ (normal 0 - 45). Se diagnostica tuberculosis peritoneal y se inicia tratamiento con isoniazida, rifampicina, pirazinamida y estreptomicina, presentando adecuada respuesta clínica.

\section{DISCUSIÓN}

La tuberculosis puede afectar cualquier parte del tracto gastrointestinal, desde la boca hasta el ano, incluyendo el peritoneo y el sistema pancreatobiliar. ${ }^{1}$ El peritoneo es la sexta parte más común de compromiso extrapulmonar y se espera que su incidencia continúe en incremento por la epidemia 
de la infección por VIH. ${ }^{2}$ Las micobacterias infectan el tracto gastrointestinal a través de la vía hematógena, por ingestión de esputo infectado o por diseminación linfática. Los estudios patológicos muestran siembras peritoneales, engrosamiento del omento, úlceras transversas y fibrosis. ${ }^{3}$ Puede tener muchas presentaciones clínicas, puede ser agudo o crónico; el 40 - 70\% de los pacientes presenta fiebre, el 80-90\% dolor abdominal, 40-90\% presentan pérdida de peso y un $20 \%$ presentan alteraciones del hábito intestinal, con frecuencia simulando otras enfermedades, ${ }^{1}$ por esta razón debe considerarse dentro del diagnóstico diferencial de los pacientes con síntomas gastrointestinales, especialmente si se acompañan de ascitis. ${ }^{3}$ Existen tres formas clínicas, la forma húmeda en la cual el hallazgo predominante es la ascitis, la forma seca con predominio de adherencias y la forma fibrótica con incremento en el espesor del omento y ascitis loculada. ${ }^{3}$ La tuberculosis peritoneal es la tercera causa de ascitis, después de la cirrosis y las neoplasias. ${ }^{4}$ El principal sitio de compromiso es la región ileocecal, presentándose a menudo como una masa en el cuadrante inferior derecho como se observó al ingreso en el examen físico de la paciente. Las radiografías de tórax evidencian compromiso pulmonar solo en el 25\% de los casos, por esta razón la ausencia de hallazgos radiológicos no descarta una posible infección extrapulmonar por Mycobacterium tuberculosis, especialmente en los pacientes inmunocomprometidos. ${ }^{5}$ Los hallazgos tomográficos incluyen ascitis loculada y colecciones de líquido en la pelvis con presencia de septos delgados que pueden simular una masa ovárica ${ }^{6}$ como en el caso descrito, ocasionado dificultades para el diagnóstico diferencial con los tumores de ovario, por su presentación clínica similar. El líquido ascítico en la tuberculosis peritoneal presenta un alto contenido de proteínas y predominio de linfocitos, la identificación de las micobacterias mediante coloración para bacilos ácido alcohol resistentes solo es positiva en menos del 3\% de los casos, ${ }^{1}$ por esta razón la visualización de las lesiones en cirugía y la toma de biopsias para el estudio patológico, son de gran importancia para el diagnóstico.

El antígeno Ca-125 es una glucoproteína de alto peso molecular cuya función fisiológica es desconocida, lo expresan las células mesoteliales y las derivadas del epitelio celómico; ${ }^{7}$ se emplea como el marcador tumoral de los tumores de ovario aunque se eleva en otros tumores ginecológicos, linfomas, melanomas, hepatocarinoma, cáncer de páncreas, tumor renal, cáncer de colon y recto. También podemos encontrar elevaciones en algunas enfermedades no neoplásicas como la peritonitis bacteriana, endometriosis, enfermedad inflamatoria pélvica, miomas, cirrosis, lupus eritematoso sistémico y en general, enfermedades que causen ascitis. ${ }^{8}$

Simsek y cols. ${ }^{9}$ realizaron un estudio de casos y controles encontrando elevación del Ca-125 en el $100 \%$ de los pacientes con tuberculosis peritoneal, con una media para los valores encontrados de 316 $\mathrm{UI} / \mathrm{ml}$, por esta razón la tuberculosis peritoneal se debe considerar dentro del diagnóstico diferencial de los pacientes con elevación del CA-125.

En conclusión, el caso descrito muestra las similitudes existentes entre la presentación clínica, los hallazgos radiológicos y la elevación de marcadores tumorales como el CA-125 que existe entre la tuberculosis peritoneal y los tumores de ovario, convirtiéndose en diagnósticos diferenciales.

\section{REFERENCIAS}

1. Sharma MP, Bhatia V. Abdominal tuberculosis. Indian J Med Res 2004;120: 305-15.

2. Paustian FF. Tuberculosis of the intestine. In: Bockus HL, editor. Gastroenterology. 2nd ed. Philadelphia: WB Saunders Co; 1964. p. 311-34.

3. Tandon HD, Prakash A. Pathology of intestinal tuberculosis and its distinction from Chron's disease. Gut 1972;13:260-9.

4. Sancho Bueso T, Martínez Arija T, García García B, Rodríguez Alvarez de la Marina J, García Puig J. Antígeno CA-125 como marcador de tuberculosis peritoneal. Rev Clin Esp 2001;10:614-5.

5. Sharma AK, Agarwal LD, Sharma CS, Sarin YK. Ab- 
dominal tuberculosis in children: experience over a decade. Indian Pediatr 1993;30:1149-53.

6. Gulati MS, Sarma D, Paul SB. CT appearences in abdominal tuberculosis. A pictorial essay. Clin Imaging 1999;23:51-9.

7. O`Brien TJ, Tanimoto Hi, Konishi J, Gee M. More than 15 years of CA 125: what is known about the antigen, its structure and its function. Int J Biol Markers
1998;131:188-95.

8. Bergman JF, Bidart JM, George M, Beaugrand M, Levy VG, Bohuon C. Elevation of CA 125 in patients with benign and malign ascitis. Cancer 1987;59:213-7.

9. Simsek H, Savas MC, Kadayifci A, Tetar G. Elevated serum CA 125 concentration in patients with tuberculous peritonitis: a case control study. Am J Gastroenterol 1997:92:1174-6.

Conflicto de intereses: ninguno declarado. 